medRxiv preprint doi: https://doi.org/10.1101/2021.11.02.21265801; this version posted November 4, 2021. The copyright holder for this preprint

(which was not certified by peer review) is the author/funder, who has granted medRxiv a license to display the preprint in perpetuity.

All rights reserved. No reuse allowed without permission.

\title{
Expanding the Russian allele frequency reference via cross-laboratory data integration: insights from 6,096 exome samples
}

Yury A. Barbitoff ${ }^{1,3,4, 凶}$, Darya N. Khmelkova ${ }^{2}$, Ekaterina A. Pomerantseva ${ }^{2}$, Aleksandr V. Slepchenkov $^{3}$, Nikita A. Zubashenko², Irina V. Mironova², Vladimir S. Kaimonov ${ }^{2}$, Dmitrii E. Polev $^{1}$, Victoria V. Tsay ${ }^{1,5}$, Andrey S. Glotov ${ }^{1,4}$, Mikhail V. Aseev ${ }^{1,4}$, Oleg S. Glotov ${ }^{1,4,5}$, Arthur A. Isaev ${ }^{2}$, and Alexander V. Predeus ${ }^{3, \varangle}$

1 - CerbaLab Ltd., St. Petersburg, Russia

2 - Genetics and Reproductive Medicine Center GENETICO Ltd., Moscow, Russia

3 - Bioinformatics Institute, St. Petersburg, Russia

4 - Dpt. of Genomic Medicine, D.O. Ott Research Institute of Obstetrics, Gynaecology and Reproductology, St. Petersburg, Russia

5 - FGBE "Children's Scientific and Clinical Center for Infectious Diseases of the Federal Medical and Biological Agency", Saint Petersburg, Russia

To whom correspondence should be addressed: barbitoff@bioinf.me, predeus@bioinf.me, Bioinformatics Institute, Kantermirovskaya St. 2A, St. Petersburg, 197342, Russia

Keywords: next-generation sequencing, whole exome sequencing, allele frequency, medical genetics, Russia, Russian populations 


\begin{abstract}
The frequency of a genetic variant in a population is crucially important for accurate interpretation of known and novel variant effects in medical genetics. Recently, several large allele frequency databases, such as Genome Aggregation Database (gnomAD), have been created to serve as a global reference for such studies. However, frequencies of many rare alleles vary dramatically between populations, and population-specific allele frequency can be more informative than the global one. Many countries and regions (including Russia) remain poorly studied from the genetic perspective. Here, we report the first successful attempt to integrate genetic information between major medical genetic laboratories in Russia. We construct an expanded reference set of genetic variants by analyzing 6,096 exome samples collected in two major Russian cities of Moscow and St. Petersburg. An approximately tenfold increase in sample size compared to previous studies allowed us to identify genetically distinct clusters of individuals within an admixed population of Russia. We show that up to 18 known pathogenic variants are overrepresented in Russia compared to other European countries. We also identify several dozen high-impact variants that are present in healthy donors despite either being annotated as pathogenic in ClinVar or falling within genes associated with autosomal dominant disorders. The constructed database of genetic variant frequencies in Russia has been made available to the medical genetics community through a variant browser available at http://ruseq.ru.
\end{abstract}




\section{Introduction}

Next-generation sequencing (NGS) has become a de facto standard tool in molecular diagnostics of Mendelian disorders. The advances in the field have been drastic; for example, whole genome sequencing (WGS) screening of all newborns is currently deployed in some countries (https://www.genomicsengland.co.uk/public-dialogue-genomics-newborn-screening/). However, despite rapid introduction of NGS into research and clinical practice, only about $42 \%$ of all patients with suspected genetic pathology receive a definitive molecular diagnosis from the trio-based WGS analysis (reviewed in (Wright, FitzPatrick, and Firth 2018)). There can be many explanations for the less-than-perfect diagnostic record of NGS-based approaches. However, most researchers agree that our ability to predict the effects of individual variants on human health is currently very limited (Biesecker and Green 2014).

One of the most significant advances of our ability to assess the variant effects came from global resequencing projects such as 1000 Genomes project (Auton et al. 2015), the Genome Aggregation Database (gnomAD), or National Heart, Blood, and Lung Institute (NHLBI) TopMed (Bick et al. 2020). A simple argument that many variants previously listed as pathogenic are found in healthy individuals in too high a frequency to cause a Mendelian disorder has become the most powerful tool of reducing false positive variant-phenotype associations (Lek et al. 2016). To this end, the information about the population allele frequency (AF) is currently broadly used for variant interpretation in clinical practice. All of the modern variant interpretation strategies and guidelines, such as the American College of Medical Genetics and Genomics (ACMG) guidelines (Richards et al. 2015), Russian variant interpretation guidelines (Ryzhkova et al. 2018), or the Sherloc guidelines (Nykamp et al. 2017) use AF in healthy populations to classify variant effects, which becomes especially critical for autosomal dominant (AD) diseases.

While global allele frequency databases remain widely useful, the additional value of population-specific reference databases was recently highlighted. Even in the original ExAC publication, Lek et al. have shown that filtering of candidate variants using the maximum allele frequency across populations substantially decreases the number of potentially disease-causing variants observed in an individual exome sample. Recently, a substantial effort of the genomic community has been directed at the creation of more diverse and inclusive populational reference as well as resources covering diverse ethnic and racial groups (e.g. (Wong et al. 2020); for review see (Martin et al. 2018)). In many countries, nation-wide sequencing projects have been conducted, 
including Genome of the Netherlands (GoNL, (Boomsma et al. 2014)) or the Han Genome Database (PGG.Han, (Gao et al. 2020)).

The Russian population, representing over 160 nationalities and many unique subpopulations, remains one of the biggest white spots on the global map of human genomic diversity (Oleksyk, Brukhin, and O’Brien 2015). Several previous studies focused on investigating the genome-wide variation of the Russian population. These include the pilot phase of the Genomes Russia project (Zhernakova et al. 2020), an exome-based study of monogenic disease prevalence in 694 patients (Barbitoff et al. 2019), and a targeted sequencing study of 242 known disease genes in 1,658 healthy individuals from the Ivanovo region (Ramensky et al. 2021). In these works, several important aspects of the genome variation in Russian patients have been pinpointed. All of these studies, however, lack in the comprehensiveness of the analysis due to either low sample size (such as in the Genomes Russia project) or narrow set of analyzed genes (Ramensky et al. 2021).

Centralized creation of a population genomic reference could benefit from funding security and uniform approaches to sequencing and analysis; at the same time, it is associated with numerous logistical and other difficulties. Most population references were de facto obtained using the aggregation of exome and genome data across multiple sequencing centers. The success of ExAC and gnomAD has proven the efficiency and scalability of this approach (Lek et al. 2016; Karczewski et al. 2020). At the same time, such integration is a challenging task due to differences in both sequencing approaches and data analysis methods used in different laboratories.

In this study, we report the first successful integration of genetic variation data across two major Russian genetic laboratories. To enhance the reproducibility of the analysis and allow a secure and confidential collaboration between the sequencing centers, we developed a portable and reproducible computational pipeline that can be used locally at any future participant laboratory, and subsequently integrated into the global reference using a pre-set analytical and legal framework. We believe that our approach allows for resource- and time-efficient aggregation of data between sequencing centers and will greatly facilitate the construction of a reliable allele frequency reference for the Russian or understudied populations. 


\section{Materials and methods}

\section{Samples and ethics statement}

The study comprised groups of patients that were subjected to NGS-based assays for molecular diagnostics and/or genetic screening at two major private centers located in Moscow (Genetico Ltd.) and St. Petersburg (CerbaLab Ltd.). As detailed below, the dataset comprised both healthy and diseased individuals, with healthy donors comprising only $12.5 \%$ of all study participants. The "diseased" subgroup included various Mendelian or likely Mendelian and nonMendelian phenotypes (with the majority of samples having neurological and neuro-muscular disorders (see Supplementary Figure 1)); the "healthy" subgroup comprised healthy donors who were sequenced for carrier screening purposes or patients with multifactorial pathologies, such as obesity or type 2 diabetes. Nearly all patients were residents of the Russian Federation or republics of the former USSR. All participants signed informed consent for studies and processing of personal data, including medical history data. The study was performed in accordance with the Declaration of Helsinki.

\section{Exome sequencing}

DNA for sequencing was extracted either from peripheral blood samples (in the majority of cases) or from tissue samples in FFPE blocks according to the standard protocols. Sequencing was performed using either whole exome (4,819 samples; $79.0 \%)$ or clinical exome (1,277 samples, $21.0 \%$ ) capture kits. The following capture kits were used: Agilent SureSelect Human all exon V7, Agilent SureSelect Human all exon V6 + UTR, TruSeq DNA Exome (Illumina) with the $x G e n ®$ Exome Research Panel v1.0 (IDT) exome capture solution, Nimblegen (Roche) SeqCap EZ MedExome, Illumina Nextera RapidCapture, Illumina TruSeq DNA Exome, Nimblegen (Roche) Inherited Disease Panel (IDP) v2, and Illumina TruSight One. Exome libraries were prepared as described previously (Barbitoff et al. 2019; 2020). All libraries were sequenced using Illumina HiSeq 2500/4000, Illumina NovaSeq 6000, MiSeq, or MGISEQ 2000. All samples were sequenced using paired-end reads with read length varying from $75 \mathrm{bp}$ to $300 \mathrm{bp}$.

\section{Bioinformatic analysis}


For bioinformatic analysis of exome sequencing data, we developed a Docker-based analysis pipeline to ease data transfer and enable the usage of each laboratory's own computing resources. For read alignment, a faster reimplementation of the BWA mem algorithm, BWAMEM2 (Vasimuddin et al. 2019) was used. Aligned reads were sorted and indexed using samtools (Li et al. 2009); duplicate read pairs were marked using the Genome Analysis ToolKit (GATK) v4.2.1.0 (Van der Auwera et al. 2013; DePristo et al. 2011). Next, base quality score recalibration and indel realignment was performed using GATK. Pre-processed alignments were then used for variant calling with the GATK HaplotypeCaller in the ERC GVCF mode. The source code of the pipeline can be found at https://github.com/bioinf/russian_exome_pipeline/

GVCF files were then transferred between laboratories and aggregated using the GATK GEnomicsDB engine. Aggregated GVCF files were then used for joint genotyping using GATK After joint genotyping, all sample-level genotypes with a total depth of less than 10 reads were set to missing, and variants with $\mathrm{AC}=0$ were excluded. The variant callset was then annotated using the Ensembl Variant Effect Predictor (VEP) v104 with the RefSeq cache file for the corresponding reference genome assembly. After variant annotation, Variant Quality Score Recalibration (VQSR) filtering approach was applied; variants with truth sensitivity score between 90.0 and 99.6 (for SNPs) and 90.0 and 99.3 (for indels) were marked as medium confidence; variants with sensitivity values > 99.6 (for SNPs) and 99.3 (for indels) were excluded as low-confidence calls. The resulting multi-sample VCF file was used for variant quality control, filtering and statistical analysis (see below).

\section{Quality control and statistical analysis}

Variant quality control and further statistical analysis of allele frequencies was performed using the BROAD Institute Hail statistical genetics library v0.2.63-cb767a7507c8 (https://hail.is/). First, sample-level quality control was performed using the built-in Hail functionality. Four main sample-level metrics were used: heterozygous to non-reference homozygous variant ratio (het/hom), transition-to-transversion ratio (Ti/Tv), insertion-to-deletion ratio, and mean persample genotype quality (GQ). The following filtering criteria were applied: $1.3<$ het/hom $<2.2$, $2.35<\mathrm{Ti} / \mathrm{Tv}<2.65 ; 0.7<\mathrm{i} / \mathrm{d}<0.9 ; \mathrm{GQ}>30$. Samples not meeting any of these criteria were removed from further analysis. We also excluded samples with less than $80 \%$ of common CDS 
bases covered at least 10x. In total, 583 samples were removed due to not passing the aforementioned filtering criteria.

After sample-level QC, we went on to filter out relatives and/or individuals with high genetic distance from the rest of the population. To do so, we first selected common (MAF > 5\%) variants that had a high (> 99\%) variant call rate across all pass-QC samples. Next, we calculated kinship statistic using the built-in Hail functionality. A maximum independent set of unrelated samples was computed using the kinship statistic value of 0.250 , removing additional 291 samples.

For principal component analysis of individual genotypes, we performed HWE-normalized PCA using the built-in Hail functionality. Samples were clustered into three clusters using $k$-means clustering algorithm in the space of first 10 principal components. Allele frequencies in each cluster were determined using Hail built-in aggregation functions.

The analysis of allele frequency correlations was performed using Python v3.9 with numpy, scipy, and pandas packages. Pearson's correlation coefficient $\left(r^{2}\right)$ was computed using a subset of variants with high (> 95\%) call rate across all samples.

Variant overrepresentation was evaluated by computing a binomial $p$-value as follows:

$$
p \sim B(n, N, q)
$$

where $n$ is the observed alternative allele count in the sample; $N$ is the total allele number at a given variant site, $q$ is the alternative allele frequency in the gnomAD non-Finnish European population, and $B$ denotes the binomial distribution PDF. Only variants reported as pathogenic in ClinVar with no conflicting interpretations were used for overrepresentation testing. All variants were annotated with the inheritance pattern associated with the corresponding gene (using the OMIM catalog data) and gene-level constraint (loss-of-function variant observed to expected ratio upper fraction, LOEUF) values from gnomAD v2.1.1. Lists of variants identified as overrepresented and/or uniquely present in the dataset were manually curated to exclude misannotated variants.

\section{Data availability}


The resulting allele frequency dataset is available through an interactive web browser at http://ruseq.ru/. To access the full VCF file with variant frequencies please contact the authors using the form available on the website.

\section{Results}

\section{Multicenter approach to the creation of Russian coding allele frequency database}

To this day, the most common approach of obtaining reliable allele frequency information relies on uniform processing of genetic data generated by multiple sequencing centers and genomic laboratories. At the same time, to perform such an integration in a centralized manner requires collection of a huge amount of data, which is very intensive in terms of both time and computing resources, and also potentially requires sharing sensitive or protected information. A possible way to circumvent these difficulties is to run the identical initial steps of data analysis in a distributed manner, and then aggregate the results at the level of per-sample variant calls. Cohort variant calling methods, such as ones provided in the GATK (DePristo et al. 2011) or GLnexus (Yun et al. 2021) packages, are streamlined to process large numbers of per-sample variant calls facilitated by the usage of specialized GVCF format.

Given these developments, efficient integration of data between sequencing centres would only require a reproducible and transferable implementation of the variant calling pipeline. This goal can be met by including all the software dependencies and code into a Docker image that can then be distributed across participating laboratories (Figure 1). In this work, we have constructed such a containerized version of a bioinformatic pipeline for data analysis based on the BWA and GATK HaplotypeCaller software. During the pilot phase of the project, this pipeline was applied in two independent sequencing centers located in two major cities of the Russian Federation, Moscow ("Lab 1") and St. Petersburg ("Lab 2"); and overall of 6,096 samples were processed in these two laboratories (4,075 - in Lab 1 and 2,021 - in Lab 2). Aggregation of variant calls across this set of samples allowed for a nearly ten-fold expansion of a previous exome-level study which included 694 samples (Barbitoff et al. 2019). The majority of samples came from patients with diagnosed monogenic disorders $(5,276)$, at the same time, as many as 820 samples were healthy 
medRxiv preprint doi: https://doi.org/10.1101/2021.11.02.21265801; this version posted November 4, 2021. The copyright holder for this preprint (which was not certified by peer review) is the author/funder, who has granted medRxiv a license to display the preprint in perpetuity.

All rights reserved. No reuse allowed without permission.

donors or patient relatives, a subgroup that would be extensively used for disease allele prevalence analysis described below.

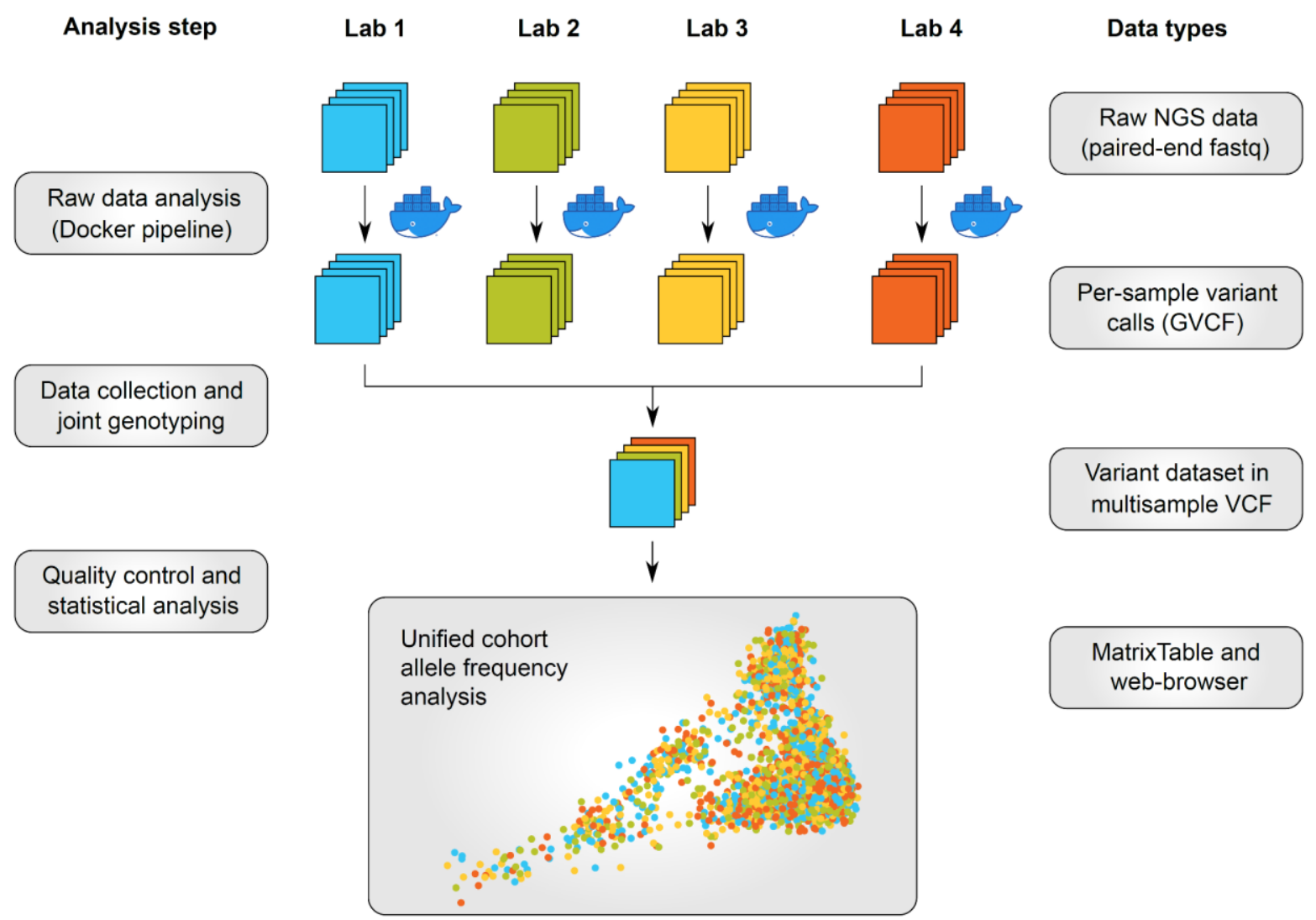

Figure 1. A cross-laboratory data integration strategy used for creation of an expanded reference set of allele frequencies in Russian exomes. The first steps of the data analysis, including read alignment and variant calling, are performed using a Docker-based pipeline in each laboratory, avoiding the need for raw data transfer and allowing for a distributed computing process. Variant calls in GVCF format are then aggregated, and joint genotyping is performed to obtain the final variant dataset.

Following initial data aggregation and genotyping, the dataset was subjected to extensive sample- and variant-level quality control (see Materials and Methods for more details), leaving 5,268 samples and 2,092,456 variant sites. Of these, 349,811 variant sites overlapped with the ones reported in our previous publication (Barbitoff et al. 2019). Out of all variants, $75.7 \%$ were known (found in the latest dbSNP build), and $24.3 \%(509,409)$ were novel. In total, 1,459,530 variants $(69.8 \%)$ were either non-coding or silent coding variants, 579,974 (27.7\%) were missense mutations or other moderate-impact variants, and 52,952 (2.5\%) variants were putative loss-of- 
function $(\mathrm{pLoF})$ variants. Similarly to previous findings, rare and protein-damaging variants were greatly overrepresented among the novel variants. For example, only $23.6 \%$ of non-coding and silent coding variants were novel compared to as much as $41.3 \%$ of all pLoF variants. Likewise, $89.6 \%(1,875,600)$ of all variant sites were rare (MAF < $1 \%$ in the total sample) compared to as much as $99.2 \%(505,427)$ of novel variants. Finally, allele frequencies of variants in samples of each of the participating parties showed a perfect correlation $\left(r^{2}=0.999\right.$, Supplementary Figure S2).

\section{Analysis of the fine genetic structure of the admixed Russian population}

In contrast to all previous genomic studies (Zhernakova et al. 2020; Barbitoff et al. 2019; Ramensky et al. 2021), our analysis includes a diverse set of admixed samples that can allow us to investigate the fine structure of the present-day Russian population. Indeed, principal component analysis of the individual genotypes identified several distinct clusters of samples. Presence of these clusters could not be explained by place of sample collection (Figure 2a), sequencing platform and/or exome kit (Supplementary Figures S3-S4). Given this observation, we went on to group the individuals into three clusters using the unsupervised k-means algorithm. The three clusters significantly differed in size and shape. The first cluster that was dubbed "heel" was the densest and contained 4,429 (84.1\%) samples. The second cluster ("ankle") was more sparse and smaller in size, containing 623 (11.8\%) samples. Finally, the third cluster ("toes") was the smallest

and the most heterogeneous, spreading over the first principal component axis (Figure 2b), and comprising 216 (4.1\%) individuals. 

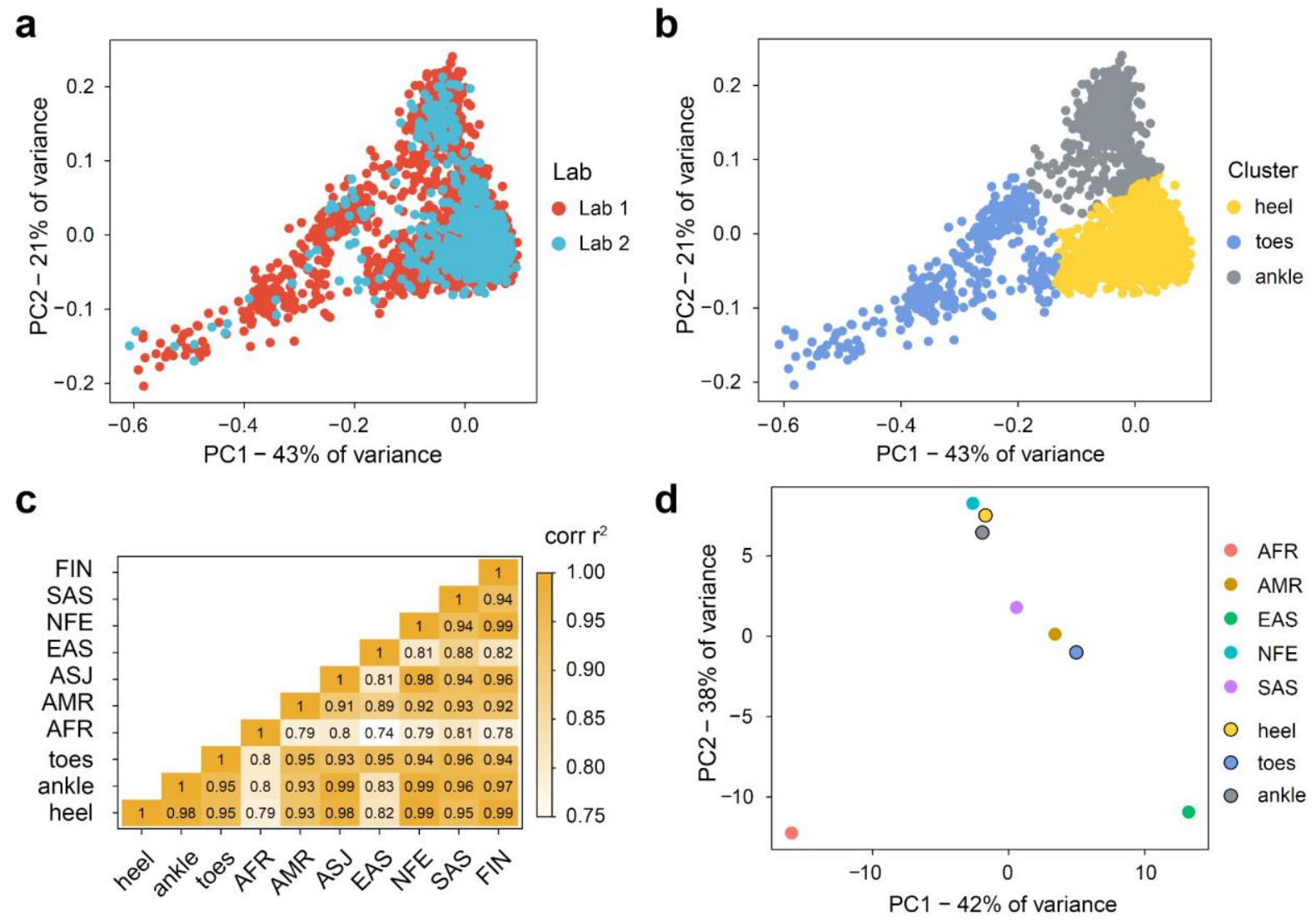

Figure 2. Analysis of the sub-structure of the admixed Russian population. (a, b) Principal component analysis of the genotypes colored by the genetic centre (a) or the results of k-means clustering in the space of first 10 principal components (b). (c) A heatmap showing Pearson's correlation between common variant allele frequencies in gnomAD ancestral groups and three clusters of individuals identified by $k$-means in (b). (d) Principal component analysis of allele frequencies of common variants in gnomAD and three clusters of individuals identified by $k$-means in (b).

Having identified the three distinct subgroups of samples, we then asked which of the global ancestral groups are closest to these clusters. To answer this question, we first analyzed the correlation between common variant allele frequencies between each cluster and the main seven populations of gnomAD (African (AFR), Ad Mixed American (AMR), Ashkenazi Jewish (ASJ), East Asian (EAS), European - Finnish (FIN) and non-Finnish (NFE), and South Asian (SAS)). The main cluster of Russian individuals ("heel") had the greatest correlation with the gnomAD NFE group $\left(r^{2}=0.991\right)$, closely followed by the Finnish population $\left(r^{2}=0.988\right)$. At the same time, the second ("ankle") cluster had a high degree of AF correlation with the NFE group $\left(r^{2}=0.987\right)$, was closer to Ashkenazi Jews than to the Finnish individuals from gnomAD $\left(r^{2}=0.987\right.$ and $r^{2}=$ 0.980 , respectively), and had a much higher AF correlation with both EAS and SAS. Finally, the 
third ("toes") cluster showed the greatest correlation with the SAS subpopulation from gnomAD $\left(r^{2}=0.975\right)$, and was equally distinct from EAS and NFE $\left(r^{2}=0.964\right.$ for both, Figure $\left.2 \mathrm{c}\right)$. In addition to the observations made by the analysis of AF correlation, we performed principal component analysis of common variant allele frequencies using data from the three clusters and five major gnomAD ancestral groups (AFR, AMR, EAS, NFE, and SAS). This analysis showed that, as expected, both the "heel" and "ankle" clusters were close to the NFE group, with the "ankle" found to be closer to SAS/EAS. At the same time, the heterogeneous "toes" cluster was much more distant and appeared closer to the gnomAD SAS (Figure 2d, Supplementary Figure $\mathrm{S} 2)$.

Given these observations, we conclude that the first cluster represents the individuals of European ancestry, i.e. native residents of the Central and Northwest Russia; the second cluster represents Southern Russia and Northern Caucasus populations, while the third cluster corresponds to patients originating from the Siberian regions and/or Asian republics of the former USSR. These assumptions were further validated by the available patient information from both sequencing centers (data not shown).

\section{Identification and validation of overrepresented pathogenic and benign variants}

Local allele frequency reference compendia are essential for two major applications: (i) to characterize the spectrum of highly prevalent clinically significant variants that can be included into early screening programs; and (ii) to identify potentially clinically significant variants that are too common in the local population to be interpreted as disease-causing. The expanded set of allele frequencies obtained in this study may aid in solving both of these critically important tasks.

Before identifying overrepresented variants in our data, we examined allele frequencies of pathogenic variants that are known to be present at high frequency in Russia. These include (i) rs113993960 variant in CFTR leading to the deletion of a crucial F508 residue of the CFTR protein, and (ii) rs80338939 variant in GJB2 linked to hearing loss and deafness (Abramov et al. 2016). In concordance with previous genetic epidemiology studies, both of these variants were present at a very high frequency in our dataset ( $\mathrm{AF}=0.0052$ for rs113993960; $\mathrm{AF}=0.0156$ for rs80338939). These results validate earlier observations and show that the constructed allele frequency reference accurately captures some of the known clinically significant genetic variation in Russia. 
Nearly two dozen prevalent and overrepresented pathogenic variants were reported in the two major sequencing-based studies of the Russian population (Barbitoff et al. 2019; Ramensky et al. 2021). We first questioned if the variants identified as overrepresented in earlier studies are also confirmed in our dataset. Overall, 22 variants that were reported in these two publications: 14 in Barbitoff et al. and 10 in Ramensky et al., with 2 overlapping variants. Of these, overrepresentation of 10 variants was successfully validated using the healthy donor subset, and of 15 - in the complete set of 5,268 samples (Supplementary Table S1).

We next went on to identify all variants that showed overrepresentation in our dataset. To this end, we computed the binomial overrepresentation p-value for all variants that were reported as pathogenic in ClinVar with no conflicting interpretations. Multiple submissions to ClinVar were required to include a variant into the analysis. As a result, 19 disease alleles were identified as overrepresented in the healthy donor subset (Table 1). These included both known high-frequency variants, such as the rs5030654 variant in $P A H$ and the rs1555287300 in ATP7B linked to phenylketonuria and Wilson's disease respectively, and variants that have not been previously reported as overrepresented. The latter category included such variants as rs554847663 in OTOG linked to autosomal recessive deafness, and variant rs119473033 in SMARCAL1 causing Schimke immuno-osseous dysplasia. High incidence of these variants has not been previously noted, and no estimates of the corresponding disease frequency are available for the Russian population. 
medRxiv preprint doi: https://doi.org/10.1101/2021.11.02.21265801; this version posted November 4, 2021. The copyright holder for this preprint (which was not certified by peer review) is the author/funder, who has granted medRxiv a license to display the preprint in perpetuity.

All rights reserved. No reuse allowed without permission.

Table 1. Known pathogenic variants present at high frequency in the expanded allele frequency reference of the Russian population.

\begin{tabular}{|c|c|c|c|c|c|c|}
\hline Variant ID & Gene & $\begin{array}{l}\text { gnomAD } \\
\text { NFE AF }\end{array}$ & $\begin{array}{l}\text { Allele } \\
\text { count }\end{array}$ & $\begin{array}{c}\text { RUSeq } \\
\text { AF* }^{*}\end{array}$ & p-value & Disease \\
\hline rs200482683 & NPHS2 & $0.02 \%$ & 5 & $0.35 \%$ & 2.07E-05 & Nephrotic syndrome \\
\hline rs549794342 & $N E B$ & $0.05 \%$ & 8 & $0.56 \%$ & $5.86 \mathrm{E}-07$ & Muscular dystrophy \\
\hline rs119473033 & SMARCAL1 & $0.01 \%$ & 4 & $0.28 \%$ & $4.62 \mathrm{E}-05$ & $\begin{array}{l}\text { Schimke immuno- } \\
\text { osseous dysplasia }\end{array}$ \\
\hline rs775288140 & COL7A1 & $0.00 \%$ & 3 & $0.21 \%$ & 8.84E-06 & $\begin{array}{c}\text { Recessive dystrophic } \\
\text { epidermolysis } \\
\text { bullosa }\end{array}$ \\
\hline rs777686211 & CPLANE1 & $0.02 \%$ & 4 & $0.29 \%$ & $3.41 \mathrm{E}-04$ & Nephronophthisis \\
\hline rs104893924 & $S L C 26 A 2$ & $0.02 \%$ & 5 & $0.35 \%$ & $2.91 \mathrm{E}-05$ & $\begin{array}{l}\text { Osteochondrodysplas } \\
\text { ia }\end{array}$ \\
\hline rs386834233 & $B C K D H B$ & $0.04 \%$ & 5 & $0.35 \%$ & $2.83 \mathrm{E}-04$ & $\begin{array}{l}\text { Maple syrup urine } \\
\text { disease }\end{array}$ \\
\hline rs782316919 & SURF1 & $0.02 \%$ & 4 & $0.28 \%$ & 7.59E-05 & Cerebellar ataxia \\
\hline rs554847663 & $O T O G$ & $0.08 \%$ & 6 & $0.46 \%$ & $5.86 \mathrm{E}-04$ & $\begin{array}{l}\text { Intellectual disability } \\
\text { and autosomal } \\
\text { recessive deafness }\end{array}$ \\
\hline rs371720347 & STAC3 & $0.01 \%$ & 3 & $0.21 \%$ & $1.06 \mathrm{E}-04$ & $\begin{array}{c}\text { Bailey-Bloch } \\
\text { congenital myopathy }\end{array}$ \\
\hline rs5030858 & PAH & $0.15 \%$ & 10 & $0.70 \%$ & $7.45 \mathrm{E}-05$ & Phenylketonuria \\
\hline rs76151636 & $A T P 7 B$ & $0.13 \%$ & 9 & $0.63 \%$ & $1.26 \mathrm{E}-04$ & Wilson disease \\
\hline rs36209567 & $F 7$ & $0.10 \%$ & 7 & $0.49 \%$ & 8.87E-04 & Abnormal bleeding \\
\hline rs200389141 & $B L M$ & $0.03 \%$ & 5 & $0.35 \%$ & $6.55 \mathrm{E}-05$ & Bloom syndrome \\
\hline rs375470378 & $G A A$ & $0.03 \%$ & 4 & $0.28 \%$ & $7.21 \mathrm{E}-04$ & $\begin{array}{c}\text { Glycogen storage } \\
\text { disease }\end{array}$ \\
\hline rs387906455 & $F 8$ & $0.00 \%$ & 3 & $0.21 \%$ & $9.34 \mathrm{E}-07$ & $\begin{array}{l}\text { Hereditary factor } \\
\text { VIII deficiency } \\
\text { disease }\end{array}$ \\
\hline
\end{tabular}

* - AF is given with reference to the healthy donor subgroup. 
Having characterized the spectrum of overrepresented pathogenic alleles in our dataset, we then went on to identify potentially clinically significant variants that are present in healthy patients but are not found in gnomAD. We began by identifying known pathogenic variants missing from gnomAD v2.1.1 data that are reported in the ClinVar database. In total, we discovered 72 such variants, with 25 of them located in genes with autosomal-dominant disease inheritance (Supplementary Table S2). In addition to these variants, we also searched for potentially clinically significant variants that are absent from gnomAD but are present in the healthy subgroup. In total, more than 100 putative loss-of-function (pLoF) variants present in healthy patients were identified. Of these, 27 variants localized in genes with high degree of evolutionary conservation according to gnomAD-derived metrics (pLI, LOEUF) and with known connection to autosomal dominant phenotypes. Selected variants are listed in Supplementary Table S2. Among the known and expected pathogenic variants present in our sample, the most notable examples include variant rs397516471 in the TNNT2 gene. This variant is reported as pathogenic for left ventricular noncompaction and familial restrictive cardiomyopathy; however, it is present in 1 healthy subject in our dataset who has not yet displayed any symptoms of the disease. Another notable example is variant rs1064793825 in $\mathrm{MSH} 2$ possibly causing hereditary colorectal cancer. Similarly to the case of rs397516471 variant in TNNT2, a carrier subject has also not yet displayed any symptoms of the disorder. However, in the case of both rs397516471 and rs1064793825, family history of variant carriers listed several cases of related disorders (cardiomyopathy or cancer, respectively), suggesting that the disease is likely to manifest in the near future.

Taken together, we described two important categories of genetic variants in our dataset: (i) known pathogenic variants that are present at higher frequency in Russia, and (ii) variants with presumed pathogenic effects that are too common in Russia and/or are present in the subgroup of healthy individuals. Both of these groups of variants can be used in NGS-based assays in Russia to assist variant interpretation in clinical practice. All of the variant frequency data presented here are available to the community of medical geneticists through a web-based variant portal at http://ruseq.ru. 


\section{Discussion}

As shown in multiple studies, population-adjusted allele frequency information is useful for efficient filtering of candidate pathogenic variants. For example, filtering of variants by maximum allele frequency across populations rather than global allele frequency helps to decrease the number of candidate pathogenic variants per individual genome (Lek et al. 2016). Despite the great availability of global allele frequency information in such resources as gnomAD, many nations and local populations remain poorly represented from a genetic perspective. Russia, being the world's largest country by total land area, has long remained the widest blank spot on the genetic map of the world (Oleksyk, Brukhin, and O'Brien 2015).

Three major studies have addressed the problem of limited genomic information about the Russian population. One of the studies included only healthy donors from different ethnic backgrounds (Zhernakova et al. 2020); however, this study suffered from a very low sample size that was not sufficient for clinical purposes. An earlier study from our group included 694 samples and provided the first exome-wide estimates of disease allele prevalence (Barbitoff et al. 2019). Finally, a very recent study by Ramensky et al. included 1,658 healthy controls; unfortunately, this study was based on a targeted sequencing of only 242 genes (Ramensky et al. 2021). Despite the aforementioned limitations, data obtained in previous analyses have already been used for interpretation of clinical significance of variants (Glotov et al. 2019; Maksimov et al. 2020) and population genetics analyses (e.g., (Shikov et al. 2020; Pinheiro, Sperb-Ludwig, and Schwartz 2021)). Exome-based allele frequencies have been included into several databases, including a database of BRCA1/BRCA2 gene mutations (https://oncobrca.ru/). A dramatic (nearly tenfold, 6,096 compared to 694) increase in the sample size achieved in this study brings the available allele frequency reference for the Russian population to the level of such a project as ESP6500 (Fu et al., 2012), greatly aiding clinical specialists in variant interpretation in NGS-based assays. Increase in the sample size allowed us to identify genetically distinct clusters in the admixed sample of Russian residents, which were in line with earlier studies (Zhernakova et al. 2020). At the same time, existence of distinct clusters imply that different genetic disease risk factors are present in different geographical regions of Russia, which is well known to the Russian medical genetic community. This finding comes as no surprise, but predicates the need for further expansion and diversification of the dataset. 
As described in the previous section, we have successfully validated some of the earlier observations regarding overrepresentation of disease alleles in the Russian population. Out of 22 disease-associated variants that were identified in (Barbitoff et al. 2019; Ramensky et al. 2021) we confirmed overrepresentation of 14 variants $(63.7 \%)$, and identified 10 novel pathogenic alleles that have greater incidence in the Russian Federation compared to other European populations (gnomAD NFE group). For several of the identified variants, their increased prevalence in Russia has been noted in gene-level studies (Abramov et al. 2015; Balashova et al. 2020). For other variants, such as the rs554847663 variant in $O T O G$, no previous reports were published. Hence, these variants represent novel candidates that are worth looking into in further genetic epidemiology studies. In addition to the overrepresented variants, we find a limited set of variants in $\mathrm{AD}$ genes that are annotated as pathogenic in ClinVar and linked to severe disorders but are present in healthy control individuals in Russia (Supplementary Table S2). This information is important for clinical interpretation of such variants, especially in the Russian population.

While the current sample size allows for more unbiased conclusions regarding the genetic structure of the Russian population, we can still expect a large number of rare genetic variants in the rest of the population that were not covered by our analysis. Recent studies have shown that mutational saturation can only be achieved by integrating hundreds of thousands of samples (Agarwal and Przeworski 2021). Hence, further aggregation of data from sequencing centers across Russia, sequencing of more healthy donors, and inclusion of patients from distinct regions (such as in the initial design of the Genomes Russia project (Oleksyk, Brukhin, and O'Brien 2015)) are all required to fully characterize the genetic variation spectrum of present-day Russia.

\section{Acknowledgements}

We want to thank all the patients who agreed to provide their data for population analysis. We also thank Mrinal Vashisth and Mary K. Futey for their help with development of the data analysis pipeline. Statistical analysis and expert review of the results was supported by the Systems Biology Fellowship to Y.A.B. the Presidential Fellowship for Young Scientists (grant no. SP4503.2021.4) to Y.A.B., and D.O. Ott Research Institute of Obstetrics, Gynaecology and Reproductology, project 558-2019-0012 (AAAA-A19119021290033-1) of FSBSI. 


\section{Conflict of interest}

Analysis of NGS data and the construction of the catalogue of allele frequencies was funded jointly by the Genetics and Reproductive Medicine Center GENETICO Ltd. and CerbaLab Ltd. according to the partnership agreement dated 25.10.2021. All rights to the data and resources generated during the work are reserved to the aforementioned parties.

\section{References}

Abramov, DD, MV Belousova, VV Kadochnikova, AA Ragimov, and D Yu Trofimov. 2016. "Carrier Frequency of GJB2 and GALT Mutations Associated with Sensorineural Hearing Loss and Galactosemia in the Russian Population." Bulletin of Russian State Medical University, no. 6.

Abramov, DD, VV Kadochnikova, EG Yakimova, MV Belousova, AV Maerle, IV Sergeev, AA Ragimov, AE Donnikov, and DY Trofimov. 2015. "High Carrier Frequency of CFTR Gene Mutations Associated with Cystic Fibrosis, and PAH Gene Mutations Associated with Phenylketonuria in Russian Population." Bulletin of RSMU 4: 32-35.

Agarwal, Ipsita, and Molly Przeworski. 2021. "Mutation Saturation for Fitness Effects at Human CpG Sites.” Preprint. Evolutionary Biology. https://doi.org/10.1101/2021.06.02.446661.

Auton, Adam, Gonçalo R. Abecasis, Steering committee, David M. Altshuler, Richard M. Durbin, Gonçalo R. Abecasis, David R. Bentley, et al. 2015. "A Global Reference for Human Genetic Variation.” Nature 526 (7571): 68-74. https://doi.org/10.1038/nature15393.

Balashova, Mariya S., Inna G. Tuluzanovskaya, Oleg S. Glotov, Andrey S. Glotov, Yury A. Barbitoff, Mikhail A. Fedyakov, Diana A. Alaverdian, et al. 2020. "The Spectrum of Pathogenic Variants of the ATP7B Gene in Wilson Disease in the Russian Federation." Journal of Trace Elements in Medicine and Biology 59 (May): 126420. https://doi.org/10.1016/j.jtemb.2019.126420.

Barbitoff, Yury A., Dmitrii E. Polev, Andrey S. Glotov, Elena A. Serebryakova, Irina V. Shcherbakova, Artem M. Kiselev, Anna A. Kostareva, Oleg S. Glotov, and Alexander V. Predeus. 2020. "Systematic Dissection of Biases in Whole-Exome and Whole-Genome Sequencing Reveals Major Determinants of Coding Sequence Coverage." Scientific Reports 10 (1): 2057. https://doi.org/10.1038/s41598-020-59026-y.

Barbitoff, Yury A., Rostislav K. Skitchenko, Olga I. Poleshchuk, Anton E. Shikov, Elena A. Serebryakova, Yulia A. Nasykhova, Dmitrii E. Polev, et al. 2019. "Whole-exome Sequencing Provides Insights into Monogenic Disease Prevalence in Northwest Russia." Molecular Genetics \& Genomic Medicine 7 (11). https://doi.org/10.1002/mgg3.964.

Bick, Alexander G., Joshua S. Weinstock, Satish K. Nandakumar, Charles P. Fulco, Erik L. Bao, Seyedeh M. Zekavat, Mindy D. Szeto, et al. 2020. "Inherited Causes of Clonal Haematopoiesis in 97,691 Whole Genomes." Nature 586 (7831): 763-68. https://doi.org/10.1038/s41586-020-2819-2. 
Biesecker, Leslie G., and Robert C. Green. 2014. "Diagnostic Clinical Genome and Exome Sequencing." New England Journal of Medicine 370 (25): 2418-25. https://doi.org/10.1056/NEJMra1312543.

Boomsma, Dorret I, Cisca Wijmenga, Eline P Slagboom, Morris A Swertz, Lennart C Karssen, Abdel Abdellaoui, Kai Ye, et al. 2014. "The Genome of the Netherlands: Design, and Project Goals." European Journal of Human Genetics 22 (2): 221-27. https://doi.org/10.1038/ejhg.2013.118.

DePristo, Mark A, Eric Banks, Ryan Poplin, Kiran V Garimella, Jared R Maguire, Christopher Hartl, Anthony A Philippakis, et al. 2011. "A Framework for Variation Discovery and Genotyping Using Next-Generation DNA Sequencing Data.” Nature Genetics 43 (5): 49198. https://doi.org/10.1038/ng.806.

Gao, Yang, Chao Zhang, Liyun Yuan, YunChao Ling, Xiaoji Wang, Chang Liu, Yuwen Pan, et al. 2020. "PGG.Han: The Han Chinese Genome Database and Analysis Platform." Nucleic Acids Research 48 (D1): D971-76. https://doi.org/10.1093/nar/gkz829.

Glotov, Oleg, Elena Serebryakova, Mariia Turkunova, Olga Efimova, Andrey Glotov, Yury Barbitoff, Yulia Nasykhova, et al. 2019. "Whole-exome Sequencing in Russian Children with Non-type 1 Diabetes Mellitus Reveals a Wide Spectrum of Genetic Variants in MODY-related and Unrelated Genes." Molecular Medicine Reports, October. https://doi.org/10.3892/mmr.2019.10751.

Karczewski, Konrad J., Laurent C. Francioli, Grace Tiao, Beryl B. Cummings, Jessica Alföldi, Qingbo Wang, Ryan L. Collins, et al. 2020. "The Mutational Constraint Spectrum Quantified from Variation in 141,456 Humans." Nature 581 (7809): 434-43. https://doi.org/10.1038/s41586-020-2308-7.

Lek, Monkol, Konrad J. Karczewski, Eric V. Minikel, Kaitlin E. Samocha, Eric Banks, Timothy Fennell, Anne H. O'Donnell-Luria, et al. 2016. "Analysis of Protein-Coding Genetic Variation in 60,706 Humans." Nature 536 (7616): 285-91. https://doi.org/10.1038/nature19057.

Li, H., B. Handsaker, A. Wysoker, T. Fennell, J. Ruan, N. Homer, G. Marth, G. Abecasis, R. Durbin, and 1000 Genome Project Data Processing Subgroup. 2009. "The Sequence Alignment/Map Format and SAMtools." Bioinformatics 25 (16): 2078-79. https://doi.org/10.1093/bioinformatics/btp352.

Maksimov, V. N., D. E. Ivanoshchuk, P. S. Orlov, A. A. Ivanova, S. K. Malyutina, S. V. Maksimova, I. A. Rodina, O. V. Khamovich, V. P. Novoselov, and M. I. Voevoda. 2020. "Next Generation Sequencing in Sudden Cardiac Death (Pilot Study)." Russian Journal of Cardiology 25 (10): 3880. https://doi.org/10.15829/1560-4071-2020-3880.

Martin, Alicia R, Solomon Teferra, Marlo Möller, Eileen G Hoal, and Mark J Daly. 2018. "The Critical Needs and Challenges for Genetic Architecture Studies in Africa." Current Opinion in Genetics \& Development 53 (December): 113-20. https://doi.org/10.1016/j.gde.2018.08.005.

Nykamp, Keith, Michael Anderson, Martin Powers, John Garcia, Blanca Herrera, Yuan-Yuan Ho, Yuya Kobayashi, et al. 2017. "Sherloc: A Comprehensive Refinement of the ACMG-AMP Variant Classification Criteria." Genetics in Medicine 19 (10): 1105-17. https://doi.org/10.1038/gim.2017.37.

Oleksyk, Taras K., Vladimir Brukhin, and Stephen J. O’Brien. 2015. “The Genome Russia Project: Closing the Largest Remaining Omission on the World Genome Map." GigaScience 4 (1): 53. https://doi.org/10.1186/s13742-015-0095-0. 
Pinheiro, Franciele C., Fernanda Sperb-Ludwig, and Ida V. D. Schwartz. 2021. "Epidemiological Aspects of Hereditary Fructose Intolerance: A Database Study." Human Mutation, September, humu.24282. https://doi.org/10.1002/humu.24282.

Ramensky, Vasily E., Alexandra I. Ershova, Marija Zaicenoka, Anna V. Kiseleva, Anastasia A. Zharikova, Yuri V. Vyatkin, Evgeniia A. Sotnikova, et al. 2021. "Targeted Sequencing of 242 Clinically Important Genes in the Russian Population From the Ivanovo Region." Frontiers in Genetics 12 (October): 709419. https://doi.org/10.3389/fgene.2021.709419.

Richards, Sue, Nazneen Aziz, Sherri Bale, David Bick, Soma Das, Julie Gastier-Foster, Wayne W. Grody, et al. 2015. "Standards and Guidelines for the Interpretation of Sequence Variants: A Joint Consensus Recommendation of the American College of Medical Genetics and Genomics and the Association for Molecular Pathology." Genetics in Medicine 17 (5): 405-23. https://doi.org/10.1038/gim.2015.30.

Ryzhkova, OP, OL Kardymon, EB Prohorchuk, FA Konovalov, AB Maslennikov, VA Stepanov, AA Afanasyev, et al. 2018. "Guidelines for the interpretation of massive parallel sequencing variants (update 2018, v2)." Meditsinskaia genetika, no. 2.

Shikov, Anton E., Rostislav K. Skitchenko, Alexander V. Predeus, and Yury A. Barbitoff. 2020. "Phenome-Wide Functional Dissection of Pleiotropic Effects Highlights Key Molecular Pathways for Human Complex Traits." Scientific Reports 10 (1): 1037. https://doi.org/10.1038/s41598-020-58040-4.

Van der Auwera, Geraldine A., Mauricio O. Carneiro, Christopher Hartl, Ryan Poplin, Guillermo del Angel, Ami Levy-Moonshine, Tadeusz Jordan, et al. 2013. "From FastQ Data to HighConfidence Variant Calls: The Genome Analysis Toolkit Best Practices Pipeline: The Genome Analysis Toolkit Best Practices Pipeline." In Current Protocols in Bioinformatics, edited by Alex Bateman, William R. Pearson, Lincoln D. Stein, Gary D. Stormo, and John R. Yates, 11.10.1-11.10.33. Hoboken, NJ, USA: John Wiley \& Sons, Inc. https://doi.org/10.1002/0471250953.bi1110s43.

Vasimuddin, Md., Sanchit Misra, Heng Li, and Srinivas Aluru. 2019. "Efficient ArchitectureAware Acceleration of BWA-MEM for Multicore Systems.” In 2019 IEEE International Parallel and Distributed Processing Symposium (IPDPS), 314-24. Rio de Janeiro, Brazil: IEEE. https://doi.org/10.1109/IPDPS.2019.00041.

Wong, Karen H. Y., Walfred Ma, Chun-Yu Wei, Erh-Chan Yeh, Wan-Jia Lin, Elin H. F. Wang, Jen-Ping Su, et al. 2020. "Towards a Reference Genome That Captures Global Genetic Diversity." Nature Communications 11 (1): 5482. https://doi.org/10.1038/s41467-02019311-w.

Wright, Caroline F., David R. FitzPatrick, and Helen V. Firth. 2018. "Paediatric Genomics: Diagnosing Rare Disease in Children." Nature Reviews Genetics 19 (5): 253-68. https://doi.org/10.1038/nrg.2017.116.

Yun, Taedong, Helen Li, Pi-Chuan Chang, Michael F Lin, Andrew Carroll, and Cory Y McLean. 2021. "Accurate, Scalable Cohort Variant Calls Using DeepVariant and GLnexus." Edited by Peter Robinson. Bioinformatics $36 \quad$ (24): $5582-89$. https://doi.org/10.1093/bioinformatics/btaa1081.

Zhernakova, Daria V., Vladimir Brukhin, Sergey Malov, Taras K. Oleksyk, Klaus Peter Koepfli, Anna Zhuk, Pavel Dobrynin, et al. 2020. "Genome-Wide Sequence Analyses of Ethnic Populations across Russia.” Genomics $112 \quad$ (1): 442-58. https://doi.org/10.1016/j.ygeno.2019.03.007. 